\title{
Think autoimmunity, breath autoimmunity, and learn autoimmunity
}

\author{
Elias Toubi ${ }^{1} \cdot$ Zahava Vadasz $^{1}$
}

Received: 17 January 2019 /Revised: 25 March 2019 / Accepted: 29 March 2019 / Published online: 12 April 2019

(C) International League of Associations for Rheumatology (ILAR) 2019

The 11th international congress on autoimmunity organized by Prof Yehuda Shoenfeld in Lisbon on May 2018 was a great celebration for auto-immunologists. More than 2000 participants from the entire world shared their views on the various aspects of autoimmunity, presented updates on issues such as pathogenesis, diagnosis, and new therapeutic approaches. The current volume of Clinical Rheumatology contains some of the presentations from this fascinating meeting, emphasizing how autoimmunity is indeed complex.

1. Dendritic cells: The role of dendritic cells (DCs) in handling antigens, including auto-antigens, is considered to be a critical phase in the pathogenesis of many immunemediated and autoimmune diseases. In his review, Slobodin G. discusses the role of DCs in the pathogenesis of ankylosing spondylitis (AS) [1]. The review focuses on the role of DCs in the initiation of immune-mediated inflammation by increasing the production of proinflammatory cytokines such as IL-17. In this respect, the ability of DCs in stimulating CD4 T cells is dependent in part on the proper engagement of HLA-B27 with the costimulatory molecule CD86. When monocyte-derived CD14-CD40+HLA DR+CD86+DCs from HLA-B27+ patients with spondyloarthritis were assessed, they were found to have a markedly decreased capacity to stimulate $\mathrm{T}$ cells when compared with healthy controls. In this respect, both splenic DCs of HLA B27 transgenic rats and monocyte-derived DCs of patients with AS have demonstrated significantly lower baseline expression of class II MHC molecules. Of importance to mention, that HLA B27, normally composed of a heavy chain connected to $\beta 2$-microglobulin, was shown to form abnormal heavy chain homodimers on the surface of activated DCs.

Zahava Vadasz

zahava.vadas@b-zion.org.il

1 Division of Allergy and Clinical Immunology, Bnai-Zion Medical Center, Technion, Haifa, Israel
These have been shown to bind to different groups of immune receptors when compared with classical HLA B27 molecule, mainly KIR (killer cell immunoglobulin-like receptor) 3DL2 receptor expressed by natural killer cells (NK) and NK T cells. Ligation to HLA B27 heavy chain homodimer through KIR3DL2 has led to expansion of the pool of KIR3DL2 bearing cells as well as diminished interferon (IFN)- $\gamma$ production. In similarity with many immune cells and regulatory proteins, DCs change their phenotype depending on the state of activation and migration. Dendritic cells possess plasticity features, and depending on stimulatory conditions or cytokine milieu, they can undergo transformation and acquire stimulatory properties on one hand but a tolerogenic profile on the other $[2,3]$.

2. The association between viral infections or malignancy and autoimmune diseases: In a paper by N. Mahroum et al. the association between rheumatoid arthritis (RA) and chronic hepatitis B viral infection (HBV) was assessed, assuming that hepatitis viruses may play a role in the pathogenesis of RA. Patients with RA were compared with age and sex-matched controls regarding the proportion of HBV infection in a casecontrol study. The study was performed utilizing the medical database of Clalit Health Services. HBV infection was found to be significantly higher in RA patients compared with controls. The study indicates that viruses may contribute to the pathogenesis of autoimmune diseases and to the production of a wide range of autoantibodies [4]. Rheumatologists should improve screening and control of viruses such as $\mathrm{HBV}, \mathrm{HCV}$, and others in patients with autoimmune diseases, mainly those planned to receive immune suppressive drugs and biological agents such as anti-TNF [5]. In another paper, S. Deshayes et al. reported on the increased prevalence of malignancy in patients suffering from giant cell arteritis (GCA). Forty-nine observations of malignancies in GCA patients were followed. Solid neoplasms were reported in $33(67 \%)$ and malignant blood diseases in $16(33 \%)$. Diagnosis 
of GCA and malignancy was synchronous in 7 (14\%). The authors suggest that initial follow-up dictated by vasculitis may have led to an early identification of associated malignancy. The issue of malignancy in patients with autoimmune diseases and or related to long-term therapy with immune suppressive agents should remain under investigation [6].

3. Rare clinical aspects in autoimmune diseases: Congenital anomalies should be considered and looked for in pregnant women with SLE. M. Sousa et al. evaluated the performance of the first trimester combined screening in patients with SLE, aiming to assess the influence of pregnancy in SLE women on biochemical parameters mainly on serum chorionic gonadotropin hormone (hCG) and whether this may give an increased rate of false positivity during the first trimester. The finding of high total serum hCG values in pregnant women with a pre-existing autoimmune disease should raise a suspicion of false positive results. Correction factors should be calculated and entered in the risk algorithm, in order to avoid the rise in the number of false-positive results and consequently the overuse of invasive methods [7]. The association between cryoglobulinemia and malignancy was discussed by Tocut et al. by reporting on a case of 40 years old woman, who developed nephritic syndrome and acute renal failure 2 weeks post-partum. She harbored type I and type II cryoglobulinemia approved by renal and cutaneous biopsies. Monoclonal gammopathy of undetermined source (MGUS) was documented following a bone marrow biopsy. Despite therapy with cyclophosphamide, rituximab, plasmapheresis, and dialysis, the patient died after 8 months. It seems that an overlapping entity of type I and II cryoglobulinemia with severe multi-organ involvement is rare and usually resistant to most available therapies and is fatal [8]. Immune checkpoint inhibitor (ICI)-induced myositis is a newly emerging systemic inflammatory myopathy following the administration of ICI. In the report by Kadota H. et al. the authors review 15 cases of ICI-induced myositis, mostly developing in patients with melanoma following the treatment with anti-PD-1 alone and antiCTLA-4 alone or the combination of both. Seven of these patients died due to myocarditis, in two due to respiratory muscle paralysis and cancer progression in two. The introduction of ICI therapy is a revolutionary approach of treatment which improved the survival of advanced melanoma. On the other hand, ICI-induced myopathies are considered to be a major cause of severe morbidity and mortality the prevalence of which should be recorded $[9,10]$.

\section{Sex hormones, silicone, vaccination, and the development of autoimmunity}

The greater prevalence of autoimmunity among women during pregnancy and post-partum support the role of sex hormones in autoimmunity, namely the role of prolactin. Prolactin has a recognized immune-stimulatory effect, mainly inhibiting the negative selection of autoreactive B lymphocytes. Hyperprolactinemia is associated with increased autoimmunity, in particular with SLE severity. In a review by Borba V.V. et al., the authors discuss the link between prolactin and autoimmune diseases and motherhood. They review the issue of whether breastfeeding should be avoided among women with established SLE or in women with high risk for its development [11-13]. The association between silicone breast implants (SBI) and the development of depression, fibromyalgia, and chronic fatigue syndrome (CFS) is evaluated by Khoo T. et al. Among 30 patients who received SBI, 12 had a diagnosis of depression, 6 of fibromyalgia and 3 of CFS. This finding is significantly higher than that in patients suffering from SSc. The authors conclude that fibromyalgia and CFS are common in women following SBI, but the finding needs a bigger cohort to be assessed $[14,15]$. The immunogenicity of seasonal or pandemic influenza vaccination in patients suffering from autoimmune inflammatory rheumatoid diseases (AIRD) was evaluated evaluated by Lakota K. et al. This is especially important in patients taking immunomodulatory medications. In their study, the authors evaluated 137 vaccinated AIRD patients, and the following issues were raised: The level of protective antibodies against influenza viruses is lower in patients treated with rituximab. The possibility of autoimmune responses after multiple vaccinations should be considered $[16,17]$.

\section{Diagnostic issues in autoimmunity}

The detection of antinuclear antibodies (ANA) plays an important role in the diagnosis of autoimmune diseases such as SLE. The sensitivity and specificity of detecting ANA are assessed and compared in many different methods some of which are discussed here. Indirect immunofluorescence on Hep-2 cells (IIF) is considered a gold standard for ANA screening in clinical laboratory routine. Alternative assays other than IIF are widely performed in many laboratories in accordance with international recommendations. However, if clinical suspicion is strong and the alternative method is negative for ANA, it is recommended that IIF should be the one to approve negativity. Elia CTD Screen assay (ECS) is described pointing to its similar high specificity and sensitivity when compared with IIF. A novel Hep-2 IIF substrate (Hep-2 
ELITE/DFS70-KO) was introduced and compared with the standard Hep-2 ANA screening being useful in defining unclear mixed ANA patterns and also of high sensitivity. Rheumatoid factor (RF) is a marker for RA but is also found in many autoimmune diseases such as SLE, scleroderma, and others. In a study by Maslinska $\mathrm{M}$ et al., RF was evaluated as an immunological and prognostic factor in primary Sjogren's syndrome (pSS). RF was assessed in 75 pSS patients $(87 \%$ females and $13 \%$ males). The authors found a positive correlation between RF, ANA, and higher disease activity. It was found to be a prognostic marker but not diagnostic [18-21].

\section{Therapeutic aspects in rheumatoid arthritis}

In a meta-analysis of the literature, Venerito $\mathrm{G}$ et al. evaluated mixed treatment comparisons (MTCs) aiming to identify the most effective drugs for the treatment of early stages of rheumatoid arthritis (ERA). Fourteen studies were included in the analysis. Tofacitinib $(64.8 \%)$ followed by etanercept $(23.2 \%)$ were the drugs with the highest probability of achieving ACR50 response. Rituximab showed the highest probability of inducing ACR70 response $(52.8 \%)$. Rheumatologists should continue evaluating the various biological therapies and assess their maximal benefit in improving early rheumatoid arthritis [22, 23].

The above-listed studies are only a flavor of the many issues of autoimmunity and rheumatology which still require further clarification. Immune-mediated responses are controlled by different regulatory cells and molecules, and as such are suggested to be a therapeutic target in many autoimmune diseases. Advanced diagnostic assays for detecting antinuclear antibodies are developed and introduced into routine in most laboratories, enabling clinicians to achieve a faster and earlier diagnosis. The meeting in Lisbon was a great opportunity for fruitful discussions and the design of future collaborations between scientists from different countries.

\section{Compliance with ethical standards}

Disclosures None.

\section{References}

1. Slobodin G, Rosner I, Kessel A (2018) Dendritic cells in the pathogenesis of ankylosing spondylitis and axial spondyloarthritis. Clin Rheumatol. https://doi.org/10.1007/s10067-018-4388-4

2. Prajzlerova K, Grobelna K, Pavelka K, Senolt L, Filkova M (2016) An update on biomarkers in axial spondyloarthritis. Autoimmun Rev 15:501-509

3. Garcia-Gonzalez P, Ubilla-Olguin G, Catalan D, Schinnerling K, Aguillon JC (2016) Tolerogenic dendritic cells for reprogramming of lymphocyte responses in autoimmune diseases. Autoimmun Rev 15:1071-1080

4. Mahroum N, Tiosano S, Hejly A, Mahagna H, Waknin R, Watad A, Comaneshter D, Cohen AD, Amital H (2018) Chronic hepatitis B viral infection among RA patients - a cross-sectional control study. Clin Rheumatol. https://doi.org/10.1007/s10067-019-04448-X

5. Kobie JJ, Zheng B, Bryk P, Barnes M, Ritchlin CT, Tabechian DA, Anandarajah AP, Looney RJ, Thiele RG, Anolik JH, Coca A, Wei C, Rosenberg AF, Feng C, Treanor JJ, Lee FE, Sanz I (2011) Decreased influenza-specific B cell responses in rheumatoid arthritis patients treated with anti-tumor necrosis factor. Arthritis Res Ther 13:R209

6. Deshayes S, Liozon E, Chanson N, Sacre K, Moulinet T, Blanchard-Delaunay C, Espitia O, Versini M, Le Gallou T, Kahn JE, Grobost V, Humbert S, Samson M, Mourot Cottet R, Mazodier K, Dartevel A, Campagne J, Dumont A, Bienvenu B, Lambert M, Daumas A, Saadoun D, Aouba A, de Boysson H (2018) Concomitant association of giant cell arteritis and malignancy: a multicenter retrospective case-control study. Clin Rheumatol. https://doi.org/10.1007/s10067-018-04407-y

7. Sousa MJ, Ribeiro R, Syngelaki A, Nicolaides K (2018) First trimester combined screening in patients with systemic lupus erythematosus: impact of pre-analytical variables on risk assessment. Clin Rheumatol. https://doi.org/10.1007/s10067-019-04525-1

8. Tocut M, Rozman Z, Biro A, Winder A, Tanay A, ZandmanGoddard G (2018) The complexity of an overlap type resistant cryoglobulinemia: a case report and review of the literature. Clin Rheumatol. https://doi.org/10.1007/s10067-018-04423-y

9. Kadota H, Gono T, Shirai Y, Okazaki Y, Takeno M, Kuwana M (2018) Immune checkpoint inhibitor-induced myositis: a case report and literature review. Clin Rheumatol

10. Palmieri DJ, Carlino MS (2018) Immune checkpoint inhibitor toxicity. Curr Oncol Rep 20:72

11. Borba VV, Sharif K, Shoenfeld Y (2018) Prolactin, autoimmunity, and motherhood: when should women avoid breastfeeding? Clin Rheumatol. https://doi.org/10.1007/s10067-018-04415-y

12. Watad A, Amital H, Aljadeff G, Zandman-Goddard G, Orbach H, Shoenfeld Prolactin Y (2016) Another important player in the mosaic of autoimmunity. Isr Med Assoc J 18:542-543

13. Jara LJ, Medina G, Saavedra MA, Vera-Lastra O, Torres-Aguilar H, Navarro C, Vazquez DM, Espinoza LR (2017) Prolactin has a pathogenic role in systemic lupus erythematosus. Immun Res 65:512-523

14. Khoo T, Proudman S, Limaye V (2018) Silicone breast implants and depression, fibromyalgia and chronic fatigue syndrome in a rheumatology clinic population. Clin Rheumatol. https://doi.org/ 10.1007/s10067-019-04447-y

15. Levy Y, Ruhrman-Shahar N (2016) The ongoing debate regarding long-term safety of silicone breast augmentation rages. Isr Med Assoc J 18:754-755

16. Lakota K, Perdan-Piskmajer K, Sodin-Semrl S, Gucnik S, Subelj V, Prosenc K, Mrak Poljsak K, Tomsic M, Ambrozic A, Praprotnik S (2018) The immunogenicity of seasonal and pandemic influenza vaccination in autoimmune inflammatory rheumatic patients - a 6-month follow-up prospective study. Clin Rheumatol. https://doi. org/10.1007/s10067-019-04439-y

17. Colafrancesco S, Perricone C, Shoenfeld Y (2016) Autoimmune/ inflammatory syndrome induced by adjuvants and Sjogren's syndrome. IsrMed Assoc J 18:150-153

18. Infantino M, Shovman O, Gilburd B, Manfredi M, Grossi V, Benucci M, Damiani A, Chimenti D, Malyavantham K, Shoenfeld Y (2018) Improved accuracy in DFS pattern interpretation using a novel HEp-2 ELITE system. Clin Rheumatol. https:// doi.org/10.1007/s10067-018-04412-1 
19. Mahler M, Meroni PL, Andrade LE, Khamashta M, Bizzaro N, Casiano CA, Fritzler MJ (2016) Towards a better understanding of the clinical association of anti-DFS70 autoantibodies. Autoimmun Rev 15:198-201

20. Rigon A, Infantino M, Merone M, Iannello G, Tincani A, Cavazzana I, Carabellese N, Radice A, Manfredi M, Soda P, Afeltra A (2017) The inter-observer reading variability in antinuclear antibodies indirect (ANA) immunofluorescence test: a multicenter evaluation and review of the literature. Autoimmun Rev 16:1224-1229

21. Maslinska M, Manczak M, Kwiatkowaka B (2018) Usefulness of rheumatoid factor as an immunological and prognostic marker in PSS patients. Clin Rheumatol. https://doi.org/10.1007/s10067-019-04438-z
22. Venerito V, Lopalco G, Cacciapaglia F, Giannini M, Iannone F (2018) A Bayesian mixed treatment comparison of efficacy of biologics and small molecules in early rheumatoid arthritis. Clin Rheumatol. https://doi.org/10.1007/s10067-018-04406-z

23. Favalli EG, Raimondo MG, Becciolini A, Crotti C, Biggioggero M, Caporali R (2017) The management of first line biologic therapy failures in rheumatoid arthritis: current practice and future perspectives. Autoimmun Rev 16:1185-1195

Publisher's note Springer Nature remains neutral with regard to jurisdictional claims in published maps and institutional affiliations. 